\title{
Death receptors couple to both cell proliferation and apoptosis
}

\author{
Ralph C. Budd \\ Division of Immunobiology, Department of Medicine, Given Medical Building, D-305, \\ The University of Vermont College of Medicine, Burlington, Vermont 05405-0068, USA. \\ Phone: (802) 656-2286; Fax: (802) 656-3854; E-mail: rbudd@zoo.uvm.edu.
}

J. Clin. Invest. 109:437-442 (2002). DOI:10.1172/JCI200215077.

No one will dispute that death receptors do exactly what their name suggests, induce programmed cell death. In addition, however, several reports have recently suggested that these receptors, or their downstream regulators, may also function in certain aspects of cell growth or differentiation. These claims might be met with justifiable skepticism. Certainly there is nothing more opposite in cell fate than growth and death. Yet the signal pathways used by these two processes appear to commingle, and the eventual outcome of the signaling vector may be bent toward one result over the other by seemingly subtle balances among these components.

Here, following a brief review of the traditional signal pathways that lead from death receptor activation to apoptosis, I scrutinize the various reports of increased growth or differentiation mediated by these same receptors. I then consider newly identified signal pathways linked to death receptors that might promote growth and, finally, speculate on whether these events represent merely interesting in vitro manipulations or actual physiologically important processes.

\section{Conventional death receptor signaling}

The classical view of death receptor function is typified by Fas (CD95/APO-1), a member of the TNF receptor (TNFR) family (1). Trimerization, or more likely oligomerization of Fas, leads to formation of the deathinducing signal complex (DISC), starting with recruitment of the Fas-adapter protein FADD through their mutual death domains (DDs) (2) (Figure 1). The other end of FADD contains two death effector domains (DEDs) that recruit caspase-8 or its enzymatically inactive homologue, the Fas inhibitor FLICE inhibitory protein (FLIP). Caspase-8, the first in a series of proteases containing a critical active-site cysteine, cleaves proteins after certain aspartate residues, with specificity determined by four amino acids $\mathrm{N}$-terminal of the cleavage site $(3,4)$. Caspases are produced as proenzymes containing an $\mathrm{N}$-terminal prodomain, as well as p20 and p10 domains that form the active enzyme as a tetramer of two $\mathrm{p} 20 / \mathrm{p} 10$ heterodimers containing two active sites (5). Because the cleavage sites of the p10 and p20 subunits contain critical aspartate residues (and hence potential caspase substrate sequences), other, active caspases can clip the prodomain from inactive caspases $(5,6)$. Active caspase 8 promotes cleavage of various downstream caspases, including caspases-3, -6 , and -7. These smaller "effector" caspases lack N-terminal homoaffinity domains, such as DDs, DEDs, and CARDs (7), but they degrade various such cellular components as the nuclear lamins (8), the cytoskeletal proteins fodrin and gelsolin (9), and the inhibitor of caspase-activated DNase (ICAD), thus activating caspase-activated DNase to degrade DNA (10-12). Caspase- 8 can also cleave the $\mathrm{Bcl}-2$ homologue Bid to reveal an active truncated Bid (tBid) fragment. tBID then complexes with and inhibits Bcl-2 in the outer mitochondrial membrane, thereby inducing cell death through a mitochondrial pathway $(13,14)$.

Death of lymphocytes, or at least of T cells, can be ascribed to one of two general mechanisms. Following antigen activation, $\mathrm{T}$ cells acquire cytokine receptors and become dependent upon them for growth and survival, especially those cytokines that trigger the common cytokine receptor $\gamma$-chain. As an inflammatory response subsides, cytokines can become limiting and this withdrawal leads to apoptosis. This process does not involve Fas but, rather, is governed by the Bcl-2 family and requires Bim, a member of the so-called BH3-only subfamily of apoptotic regulators (15). A second route to $\mathrm{T}$ cell death, known as activation-induced cell death (AICD), is activated by repeated stimulation by the antigen receptor. The traditional view is that AICD results primarily from induction of the proapoptotic Fas ligand (FasL) and TNF- $\alpha$ (16). This process does not appear to be regulated by traditional $\mathrm{Bcl}-2$ family members (17), but AICD induction by superantigens is only slightly delayed in the absence of Fas (18). Clearly other factors - perhaps one or more of the BH3-only proteins - support the deletion of these cells. Recently, Bcl-3 activation of NF- $\mathrm{KB}$ was proposed as a survival signal in activated T cells (19). Nonetheless, the importance of Fas in lymphocyte homeostasis is reflected in mice and humans bearing mutations in the Fas gene, where profound lymphadenopathy and an autoimmune diathesis result $(20,21)$. 


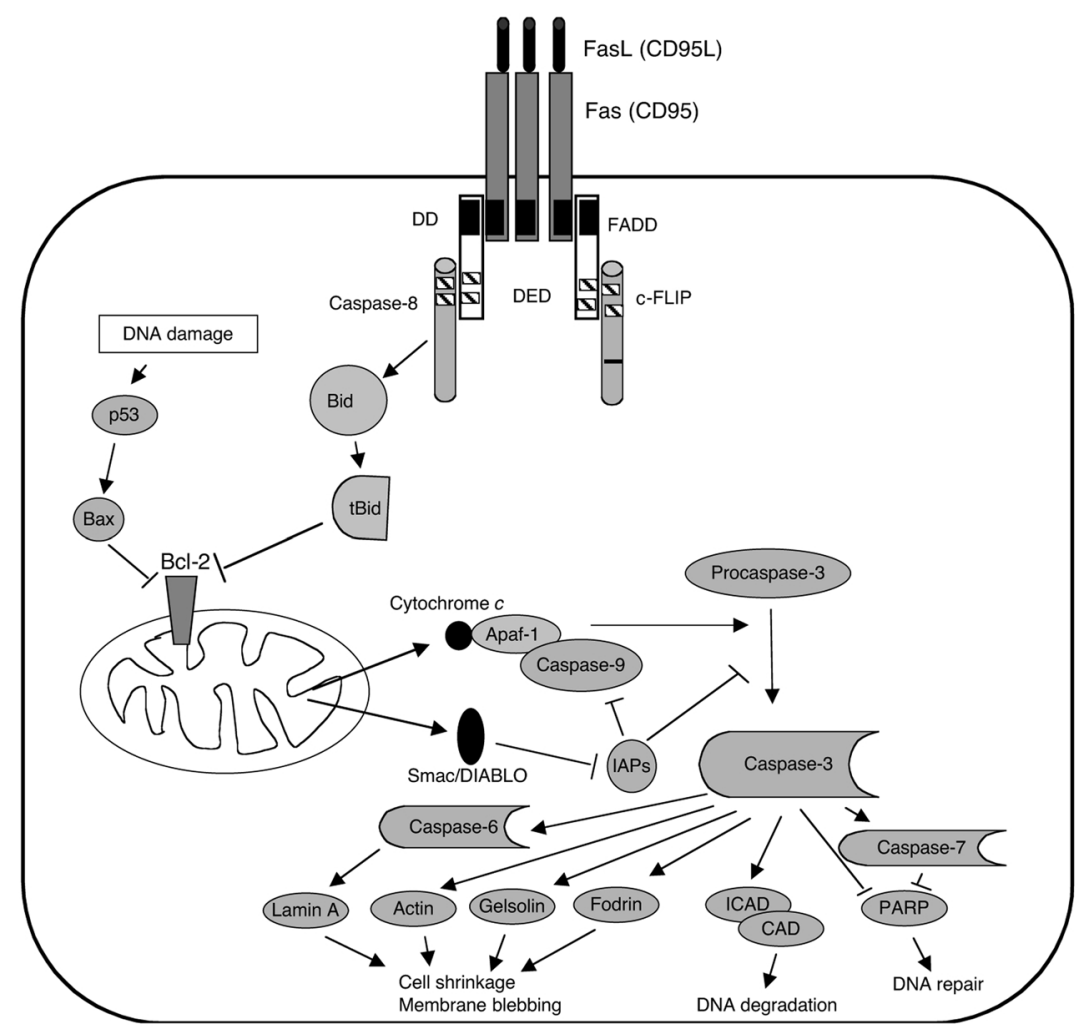

Figure 1

The death receptor pathway as typified by Fas (CD95/APO-1). Oligomerization of Fas by FasL induces recruitment of FADD to the cytoplasmic tail of Fas by their mutual DDs (black boxes). The opposite end of FADD contains a death effector domain (DED; hatched boxes) that allows recruitment of either procaspase-8 or the related protein c-FLIP, which contains a sequence change in the sequence corresponding to the active site of procaspase- 8 (black bar), rendering it enzymatically inactive. Caspase- 8 can cleave the BH3-only protein Bid, and the resulting truncated Bid (tBid) can inactivate $\mathrm{Bcl}-2$ in the mitochondrial membrane. This allows the escape of cytochrome $c$, which clusters with Apaf-1 and caspase-9 in the presence of dATP to activate caspase-9. Smac/DIABLO is also released from the mitochondria and inactivates inhibitors of apoptosis (IAPs). Active caspase-9 can cleave and activate procaspase-3 to its active form, leading to breakdown of several cytoskeletal proteins and degradation of the inhibitor of caspase-activated DNase (ICAD).

Recent findings suggest that cell death following cytokine withdrawal is dependent not on proximal caspases but, rather, on those that are more downstream. Thus, mice lacking Fas or FADD or overexpressing CrmA (which inhibits caspase-8) all inhibit Fas-induced death but do not block death by cytokine deprivation (22). The reverse is true (that is, Fas-induced death is unchanged but cytokine withdrawal is impeded) in mice transgenic for Bcl-2, in knockout animals lacking Bim, and in double knockouts lacking both Bak and $\operatorname{Bax}(22,23)$.

\section{Death receptor induction of cell growth or differentiation}

Among the first observations of increased cell growth by a death receptor was that of TNF- $\alpha$ costimulation of $\mathrm{T}$ and $\mathrm{B}$ cell growth by Lipsky and coworkers $(24,25)$. Many other reports confirm that in cell types as diverse as vascular smooth muscle (26) and dendritic (27), TNF- $\alpha$ can induce differentiation or activate cell function, if not promote cell growth. That TNF- $\alpha$ might confer opposing functions of cell growth and differentiation and death has met with less resistance than similar claims for other death receptor ligands, since two TNFR types have long been known, one (p55 TNFR1) bearing a death domain, and the other (p75 TNFR2) lacking this sequence. However, it does not appear that these two receptor types specialize in the manner that was originally supposed, since recent studies show that TNFR2 can confer signals for both death and growth in T cells (28). The levels of the TNFR-associating kinase receptor interacting protein (RIP) seem to be pivotal in this switch in T cells. RIP levels are low in resting $\mathrm{T}$ cells, which allows TNF- $\alpha$ signals to promote growth, whereas RIP levels increase with cell cycling and confer sensitivity to cell death (28). In the case of Fas signaling, despite the existence of only a single receptor (Fas), there are several instances of signals by this classically proapoptotic molecule promoting increased growth of T cells $(29,30)$, fibroblasts $(28)$, certain tumors $(31)$, hepatocytes (32), and increased differentiation of dendritic cells (33). Fas may also induce the physiological and morphological changes in cardiomyocytes seen in cardiac hypertrophy. Thus, Badorff et al. recently observed that Fas ligation of cardiac myocytes leads to phosphorylation of glycogen synthase kinase- $3 \beta$, which results in its inactivation and increased cardiac protein synthesis (34). This process is necessary for cardiac hypertrophy, and the authors found that in a model of 
cardiac overload, which ordinarily leads to hypertrophy, the hearts of Fas-deficient lpr mice fail to adapt and instead undergo dilatation. Each of these cases is worth close scrutiny to determine whether alternate interpretations of the findings are possible.

The initial suggestion that Fas might promote growth signals was inspired by work of David Lynch's group at Immunex Research and Development Corp. (Seattle, Washington, USA), showing that antibodies to Fas that were cytolytic toward tumor cells and cycling $\mathrm{T}$ cells were powerfully costimulatory for proliferation and cytokine production with $\mathrm{CD} 3$ activation of resting $\mathrm{T}$ cells (29). While there remained the possibility that this might result from the antibodies blocking ligation by endogenous FasL, many of the costimulatory anti-Fas antibodies used were cytolytic on other cells. More recently, this concern was resolved by similar findings using soluble FasL in place of antibodies. Kennedy et al. (35) showed that proliferation of CD3-activated primary human $\mathrm{T}$ cells can be augmented threefold by cross-linked, but not by uncross-linked, FasL, suggesting that oligomerization is necessary to augment proliferation, much as it is with cell death. While both studies used purified $T$ cells, it is still possible that this effect might depend on an accessory cell, for example, on a minor contamination with dendritic cells. Recently, researchers in two groups observed not only that both mouse and human dendritic cells resist FasL-induced cell death, but also that, in these cells, FasL actually induces upregulation of surface B7.1, B7.2, and MHC class II (33). Later studies have shown that dendritic cells express high levels of the Fas inhibitor FLIP (36), which may help explain the diversion of signals from cell death and toward a growth signal pathway.

In related $\mathrm{T}$ cell work, immobilized anti-Fas or soluble FasL alone has been reported to promote proliferation of $\mathrm{T}$ cells from patients with systemic lupus erythematosus (37). Furthermore, caspase inhibitors partially blocked this augmented growth. The observations are consistent with those of Suzuki and coworkers (38), who observed that Fas-Fc can attenuate the proliferation of murine $\mathrm{T}$ cells. These latter findings might indicate that blocking FasL with Fas-Fc inhibits Fas costimulation, or, as favored by the authors, that Fas-Fc can induce FasL to exert retrograde positive signals. Another report observed that anti-Fas can stimulate a nearly tenfold increase in proliferation of normal human skin fibroblasts, in a manner similar to that of TNF- $\alpha$ (39), whereas only TNF- $\alpha$ stimulates IL- 6 production. As the cultures in this study appear to have been confluent, it is unclear whether some of this effect might result from the death of some fibroblasts by anti-Fas, which would then make room for the remaining cells to re-enter cell cycling.

Two reports indicate that Fas can induce growth of tumor cells. In one study of various B cell lymphoma cell lines, whereas most were sensitized to undergo apoptosis by anti-Fas after priming with Staphylococcus aureus Cowan I (SAC) plus IL-2, one patient's lymphoma cells consistently manifested increased proliferation to antiFas (40). This lymphoma was also the only one studied in which Bcl-2 levels did not drop following treatment with SAC and IL-2. It is not clear whether Bcl-2 levels contribute to this unusual feature, since $\mathrm{Bcl}-2$ does not activate cell proliferation, and it generally does not block Fas-induced death in lymphocytes. A second study examined a variety of tumors and observed that only 4 of the 11 Fas-positive nonhematopoietic tumors were sensitive to killing by anti-Fas (31). Moreover, anti-Fas enhanced growth of 3 of the 11 tumors, including one epidermoid carcinoma, one melanoma, and one pancreatic carcinoma. In these studies, resistance to Fas and enhanced proliferation did not correlate with levels of $\mathrm{Bcl}-2$ expression.

Two further situations, somewhat more complex given their in vivo settings, also raise the possibility of Fas-induced cell growth. Biancone et al. (41) found that the subcutaneous slow release of agonistic Fas antibody from a matrix gel in mice can promote angiogenesis, activating endothelial cell infiltration and canalization, as well as a subsequent inflammatory infiltration of neutrophils in the new blood vessels. This phenomenon was dose-dependent and required interaction with Fas, as Fas-deficient $l p r$ mice did not respond in this manner. In addition, apoptotic cells were not observed at any time inside the implant or in the surrounding tissue. In another study, examining liver regeneration in mice after partial hepatectomy (32), whereas anti-Fas in vivo induced rapid hepatocyte apoptosis as observed earlier (42), the same Fas antibody increased cell cycling of hepatocytes during liver regeneration. This change in the response to anti-Fas between resting and regenerating hepatocytes is reminiscent of the differential effects of TNFR1, which also signals cell death in resting hepatocytes (43) but stimulates proliferation during liver regeneration (44). Desbarats and Newell (32) found that the change correlates with higher levels of FLIP in regenerating versus resting hepatocytes after treatment with anti-Fas, and they noted that regeneration is significantly delayed in $l p r$ mice. The source of FasL during liver regeneration was not addressed in this study, but it may be significant that athymic nude mice, bearing few T cells, manifest delayed liver regeneration (45), and that activated T cells are resident in the liver (46).

Some of these studies leave open the possibility that anti-Fas or FasL could cause the appearance of increased cell growth by actually causing cell death of some cell types, which then might allow the enhanced proliferation of the remaining cells. However, because this alternative explanation is not compelling in all these cases, the question arises as to how Fas might actually promote a cell growth signal.

\section{Death receptor signal pathways that might promote cell growth}

The involvement of death receptor signal pathways in cell proliferation suggests that other members of the DISC contribute to cell proliferation in some situations. Striking evidence for such a claim comes from work with FADD-deficient mice, which unexpectedly manifest a profound defect in $\mathrm{T}$ cell proliferation (47), leading to the proposal that caspase activity itself might be required for $T$ cell proliferation. Indeed, evidence of caspase cleavage has been observed in actively proliferating T cells, even when they were rigorously depleted of any dying cells by sorting annexin V-negative cells $(35,48)$. Although some caspase 


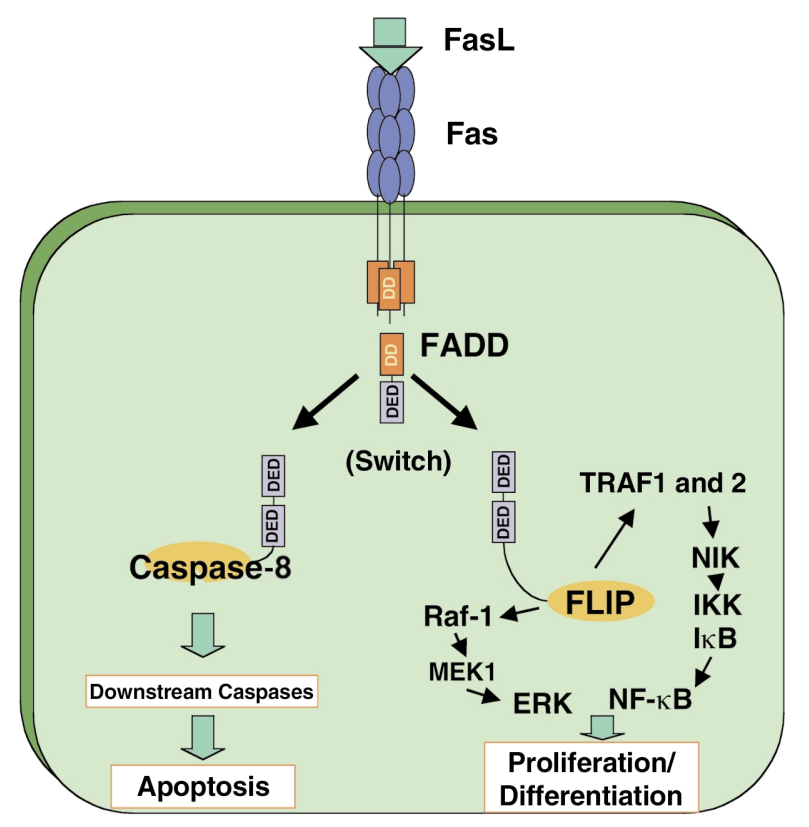

Figure 2

FLIP as a switch molecule that diverts Fas signals from cell death to proliferation. FLIP is homologous to caspase- 8 in containing two DEDs that can interact with the DED of FADD, but its caspase domain is nonfunctional. FLIP physically associates with Raf-1 and TRAF1 and TRAF2, which connect, respectively, with the ERK and NF-KB pathways involved in cell proliferation or differentiation. In this capacity, primary cells that express high levels of FLIP would not only be resistant to Fas-induced death but might also receive a proliferative signal.

blockers have been observed to at least partially inhibit $\mathrm{T}$ cell proliferation $(35,48)$, their role in the other cell growth systems induced by Fas have not been examined. Certainly, considerable cellular remodeling occurs during cell division, which requires reorganization of the cytoskeleton, and many cytoskeletal proteins, including lamins, actin, gelsolin, and fodrin, are substrates for caspases $(8,9)$. During cell division, the nuclear membrane to which lamins are attached becomes vesiculated and dispersed throughout the cytosol. Although cleavage of lamins has not been reported in this context to date, it is well established that lamins are phosphorylated, a posttranslational modification that is associated with depolymerization of the lamina into soluble oligomeric units $(49,50)$.

It should also be remembered that not all caspases serve to inactivate proteins. Indeed, the first caspase identified, IL- $1 \beta$-converting enzyme (ICE/caspase-1), cleaves IL-1 $\beta$ and IL-18 into active proinflammatory cytokines (51), which might contribute to inflammatory angiogenesis, as described above. A number of other signaling molecules $-\mathrm{c}$-Jun $\mathrm{NH}_{2}$-terminal kinase (JNK), PITSLRE kinase, protein kinase-C $\delta$, and p21activated kinase- 2 , among others $(52,53)$ - have been identified as caspase substrates, although the significance of their cleavage remains unclear.

Death receptors might also promote growth that is independent of caspases by engaging alternative signal pathways. A logical candidate in the case of Fas is FLIP. In addition to its ability to block Fas-induced death and caspase activation, FLIP can also divert signals toward pathways that connect to the mitogen-activated protein kinase extracellular signal-regulated kinase (ERK) and also to NF-KB (54). Jurkat T cells overexpressing FLIP manifest increased phospho-ERK, as well as increased phosphorylation (and hence degradation) of IKB $\alpha$, the negative regulator of NF- $\kappa B$. The link in these pathways to FLIP has been found to be the associations of FLIP with Raf-1, which activates MEK1 to activate ERK, and with TNFR associated factor 1 (TRAF1) and TRAF2, which lead to NF- $\kappa B$ activation. In T cells, the ERK and NF- $\kappa B$ pathways impinge on the IL2 promoter. Indeed, overexpression of FLIP in Jurkat cells results in increased IL-2 production (54). Furthermore, mice transgenic for FLIP in the $\mathrm{T}$ cell compartment manifest considerably higher rates of cell cycling to low-dose anti-CD3 or antigen stimulation (our unpublished observations). Knowing that many tumors, especially melanomas (55), express high levels of FLIP, as do dendritic cells (36), this might explain not only the resistance of these cells to Fas-induced death, but also the increased growth or differentiation manifested by some of them upon Fas ligation. The model emerging from these findings is thus that FLIP serves as a switch between life and death signals conferred by Fas (Figure 2), such that, when FLIP levels are low, Fas signals are biased toward recruiting caspase- 8 to the DISC, resulting in its cleavage and activation. By contrast, when FLIP levels are high, it may be preferentially recruited to the DISC, diverting signals down the ERK and NF-KB pathways.

A somewhat similar model has been proposed by Brian Seed's group for the role of the classically proapoptotic molecule RIP in TNFR2 signaling (28). These authors argue that, whereas in resting $\mathrm{T}$ cells, RIP levels are low and TNFR2 signals activate NF- $\mathrm{KB}$, IL-2-driven cell proliferation causes a rise in RIP levels, diverting TNFR2 signals toward promoting apoptosis. In a similar manner, FLIP levels decrease in cycling $\mathrm{T}$ cells and also confer susceptibility to Fas-induced death (28). These findings may collectively explain in part the resistance of resting $\mathrm{T}$ cells to apoptosis but their sensitivity to death signals upon cell cycling.

\section{In vitro versus real physiology}

It is worth recalling that genetic deficiencies of Fas, TNFR1, TNFR2, and TRAIL have not revealed any obvious abnormality of cell growth or development, outside of the immune system and hepatocyte regeneration in Fas-deficient lpr mice. At a minimum, studies on cell growth or differentiation by death receptors will tell us how certain death signal pathways dovetail, even if only briefly, with those of cell growth. There may be no further overlap in the function of growth and death receptors. On the other hand, these findings raise the possibility of a more significant overlap, possibly even a requirement for cells to enter cell cycling as part of their death spiral, at least in some cell types. Such a model would be consistent with the suggestion that premature activation of certain cell cycle genes, such as p34cdc2, is required for apoptosis (56). The similarities between certain morphological features of mitosis and apoptosis, such as nuclear membrane and chromatin condensation, bring to mind the proposed view of apoptosis as "mitotic catastrophe" (56). Viewed the other way around, apoptosis may be the more 
common default outcome of attempted cell division, whereas successful completion of the cell cycle is more the exception. And just conceivably, these collective findings may point toward more extensive linkage between the wiring of cell growth and death, suggesting that there is a considerable advantage to insuring that cells prone to bursts of rapid proliferation carry with them the requisite receptors to secure subsequent cell death.

1. Nagata, S., and Golstein, P. 1995. The Fas death factor. Science. 267:1449-1456.

2. Krammer, P.H. 1999. CD95(APO-1/Fas)-mediated apoptosis: live and let die. Adv. Immunol. 71:163-210.

3. Thornberry, N.A., et al. 1997. A combinatorial approach defines specificities of members of the caspase family and granzyme B. Functional relationships established for key mediators of apoptosis. J. Biol. Chem. 272:17907-17911.

4. Thornberry, N.A., and Lazebnik, Y. 1998. Caspases: enemies within. Science. 281:1312-1316.

5. Earnshaw, W.C., Martins, L.M., and Kaufmann, S.H. 1999. Mammalian caspases: structure, activation, substrates, and functions during apoptosis. Annu. Rev. Biochem. 68:383-424.

6. Alnemri, E.S., et al. 1996. Human ICE/CED-3 protease nomenclature. Cell. 87:171.

7. Hofmann, K. 1999. The modular nature of apoptotic signaling proteins. Cell. Mol. Life Sci. 55:1113-1128.

8. Rao, L., Perez, D., and White, E. 1996. Lamin proteolysis facilitates nuclear events during apoptosis. J. Cell Biol. 135:1441-1455.

9. Kothakota, S., et al. 1997. Caspase-3-generated fragment of gelsolin: effector of morphological change in apoptosis. Science. 278:294-298.

10. Liu, X., Zou, H., Slaughter, C., and Wang, X. 1997. DFF, a heterodimeric protein that functions downstream of caspase-3 to trigger DNA fragmentation during apoptosis. Cell. 89:175-184.

11. Enari, M., et al. 1998. A caspase-activated DNase that degrades DNA during apoptosis, and its inhibitor ICAD [erratum 1998, 393:396]. Nature. 391:43-50.

12. Sakahira, H., Enari, M., and Nagata, S. 1998. Cleavage of CAD inhibitor in CAD activation and DNA degradation during apoptosis. Nature. 391:96-99.

13. Li, H., Zhu, H., Xu, C.J., and Yuan, J. 1998. Cleavage of BID by caspase 8 mediates the mitochondrial damage in the Fas pathway of apoptosis. Cell. 94:491-501.

14. Kim, T.H., Zhao, Y., Barber, M.J., Kuharsky, D.K., and Yin, X.M. 2000. Bid-induced cytochrome $c$ release is mediated by a pathway independent of mitochondrial permeability transition pore and Bax. J. Biol. Chem. 275:39474-39481.

15. Bouillet, P., et al. 1999. Proapoptotic Bcl-2 relative Bim required for certain apoptotic responses, leukocyte homeostasis, and to preclude autoimmunity. Science. 286:1735-1738.

16. Brunner, T., et al. 1995. Cell-autonomous Fas (CD95)/Fas-ligand interaction mediates activation-induced apoptosis in T-cell hybridomas. Nature. 373:342-345.

17. Strasser, A., Harris, A.W., Huang, D.C., Krammer, P.H., and Cory, S. 1995. Bcl-2 and Fas/APO-1 regulate distinct pathways to lymphocyte apoptosis. EMBOJ. 14:6136-6147.

18. Mixter, P.F., Russell, J.Q., and Budd, R.C. 1994. Delayed kinetics of T lymphocyte anergy and deletion in lpr mice. J. Autoimmun. 7:697-710.

19. Mitchell, T.C., et al. 2001. Immunological adjuvants promote activated $\mathrm{T}$ cell survival via induction of Bcl-3. Nat. Immunol. 2:397-402.

20. Budd, R.C., Van Houten, N., Clements, J., and Mixter, P.F. 1994. Parallels in T lymphocyte development between $l p r$ and normal mice. Semin. Immunol. 6:43-50.

21. Drappa, J., Vaishnaw, A.K., Sullivan, K.E., Chu, J.-L., and Elkon, K.B. 1996. Fas gene mutation in the Canale-Smith syndrome, an inherited lymphoproliferative disorder associated with autoimmunity. N. Engl.J. Med. 335:1643-1650.

22. Strasser, A. 2001. BH3-only members of the Bcl-2 family are critical inducers of apoptosis and preclude autoimmunity. Keystone Symposium on Molecular Mechanisms of Apoptosis. January 16-22, 2001. Keystone, Colorado, USA. Abstract 003.

23. Thompson, C. 2001. The pro-apoptotic Bcl-2 family members Bak and Bax play complementary roles in blocking apoptosis. Keystone Symposium on Molecular Mechanisms of Apoptosis. January 16-22, 2001. Keystone, Colorado, USA. Abstract 472.

24. Yokota, S., Geppert, T.D., and Lipsky, P.E. 1988. Enhancement of antigen- and mitogen-induced human T lymphocyte proliferation by tumor necrosis factor-alpha. J. Immunol. 140:531-536.

25. Jelinek, D.F., and Lipsky, P.E. 1987. Enhancement of human B cell proliferation and differentiation by tumor necrosis factor-alpha and interleukin 1. J. Immunol. 139:2970-2976.
26. Beutler, B., and van Huffel, C. 1994. Unraveling function in the TNF ligand and receptor families. Science. 264:667-668.

27. Larsen, C.P., et al. 1990. Migration and maturation of Langerhans cells in skin transplants and explants. J. Exp. Med. 172:1483-1493.

28. Pimentel-Muinos, F.X., and Seed, B. 1999. Regulated commitment of TNF receptor signaling: a molecular switch for death or activation. Immunity. 11:783-793.

29. Alderson, M.R., et al. 1993. Fas transduces activation signals in normal human T lymphocytes. J. Exp. Med. 178:2231-2235.

30. Sakata, K., et al. 1998. Fas (CD95)-transduced signal preferentially stimulates lupus peripheral T lymphocytes. Eur. J. Immunol. 28:2648-2660.

31. Owen-Schaub, L.B., Radinsky, R., Kruzel, E., Berry, K., and Yonehara, S. 1994. Anti-Fas on nonhematopoietic tumors: levels of Fas/APO-1 and bcl-2 are not predictive of biological responsiveness. Cancer Res. 54:1580-1586.

32. Desbarats, J., and Newell, M.K. 2000. Fas engagement accelerates liver regeneration after partial hepatectomy. Nat. Med. 6:920-923.

33. Rescigno, M., et al. 2000. Fas engagement induces the maturation of dendritic cells (DCs), the release of interleukin (IL)-1beta, and the production of interferon gamma in the absence of IL-12 during DC-T cell cognate interaction. A new role for fas ligand in inflammatory responses. J. Exp. Med. 192:1661-1668.

34. Badorff, C., et al. 2002. Fas receptor signaling inhibits glycogen synthase kinase $3 \beta$ and induces cardiac hypertrophy following pressure overload. J. Clin. Invest. 109:373-381. DOI:10.1172/JCI200213779.

35. Kennedy, N.J., Kataoka, T., Tschopp, J., and Budd, R.C. 1999. Caspase activation is required for T cell proliferation. J. Exp. Med. 190:1891-1896.

36. Ashany, D., Savir, A., Bhardwaj, N., and Elkon, K.B. 1999. Dendritic cells are resistant to apoptosis through the Fas (CD95/APO-1) pathway. J. Immunol. 163:5303-5311.

37. Sakata, K., et al. 1998. Fas (CD95)-transduced signal preferentially stimulates lupus peripheral $\mathrm{T}$ lymphocytes. Eur. J. Immunol. 28:2648-2660.

38. Suzuki, I., Martin, S., Boursalian, T.E., Beers, C., and Fink, P.J. 2000. Fas ligand costimulates the in vivo proliferation of CD8+ T cells. J. Immunol. 165:5537-5543.

39. Aggarwal, B.B., Singh, S., LaPushin, R., and Totpal, K. 1995. Fas antigen signals proliferation of normal human diploid fibroblast and its mechanism is different from tumor necrosis factor receptor. FEBS Lett. 364:5-8.

40. Mapara, M.Y., et al. 1993. APO-1 mediated apoptosis or proliferation in human chronic B lymphocytic leukemia: correlation with bcl-2 oncogene expression. Eur. J. Immunol. 23:702-708.

41. Biancone, L., et al. 1997. Development of inflammatory angiogenesis by local stimulation of Fas in vivo. J. Exp. Med. 186:147-152.

42. Osagawara, J., et al. 1993. Lethal effects of the anti-Fas antibody in mice. Nature. 364:806-809.

43. Bradham, C.A., Plumpe, J., Manns, M.P., Brenner, D.A., and Trautwein, C. 1998. Mechanisms of hepatic toxicity. I. TNF-induced liver injury. Am. J. Physiol. 275:G387-G392.

44. Yamada, Y., Webber, E.M., Kirillova, I., Peschon, J.J., and Fausto, N. 1998. Analysis of liver regeneration in mice lacking type 1 or type 2 tumor necrosis factor receptor: requirement for type 1 but not type 2 receptor. Hepatology. 28:959-970.

45. Albrecht, J.H., Hoffman, J.S., Kren, B.T., and Steer, C.J. 1994. Changes in cell cycle-associated gene expression in a model of impaired liver regeneration. FEBS Lett. 347:157-162.

46. Ohteki, T., et al. 1992. Age-dependent increase of extrathymic T cells in the liver and their appearance in the periphery of older mice. J. Immunol. 149:1562-1570.

47. Zhang, J., Cado, D., Chen, A., Kabra, N.H., and Winoto, A. 1998. Fasmediated apoptosis and activation-induced T-cell proliferation are defective in mice lacking FADD/Mort1. Nature. 392:296-300.

48. Alam, A., Cohen, L.Y., Aouad, S., and Sekaly, R.P. 1999. Early activation of caspases during $T$ lymphocyte stimulation results in selective substrate cleavage in nonapoptotic cells. J. Exp. Med. 190:1879-1890.

49. Gerace, L., and Blobel, G. 1980. The nuclear envelope lamina is reversibly depolymerized during mitosis. Cell. 19:277-287.

50. Chaudhary, N., and Courvalin, J.C. 1993. Stepwise reassembly of the nuclear envelope at the end of mitosis. J. Cell Biol. 122:295-306.

51. Yuan, J., Shaham, S., Ledoux, S., Ellis, H.M., and Horvitz, H.R. 1993. The C. elegans cell death gene ced-3 encodes a protein similar to mammalian interleukin-1 beta-converting enzyme. Cell. 75:641-652.

52. Villa, P., Kaufmann, S.H., and Earnshaw, W.C. 1997. Caspases and caspase inhibitors. Trends Biochem. Sci. 22:388-393.

53. Cryns, V., and Yuan, J. 1998. Proteases to die for. Genes Dev. 12:1551-1570.

54 . Kataoka, T., et al. 2000. The caspase-8 inhibitor FLIP promotes activation of NF-kappaB and Erk signaling pathways. Curr. Biol. 10:640-648.

55. Irmier, M., et al. 1997. Inhibition of death receptor signals by cellular FLIP. Nature. 388:190-195.

56. Shi, L., et al. 1994. Premature p34cdc2 activation required for apoptosis. Science. 263:1143-1145. 